\title{
BlinGui: uma solução vestível de apoio a pessoas portadoras de deficiência visual na detecção de obstáculos estáticos em ambientes internos
}

\author{
Elidiane P. dos Santos ${ }^{1}$, Flávia S. Nascimento ${ }^{1,2}$, Vaninha Vieira ${ }^{1,3}$ \\ ${ }^{1}$ Programa de Pós-Graduação em Computação da Universidade Federal da Bahia (UFBA) \\ ${ }^{2}$ Instituto Federal de Educação, Ciência e Tecnologia da Bahia (IFBA) \\ ${ }^{3}$ Centro de Projetos Fraunhofer da UFBA \\ Salvador - BA - Brasil \\ \{elidiane.pereira,vaninha\}@ufba.br, flaviamsn@ifba.edu.br
}

\begin{abstract}
Frequently, visually impaired people bump into objects during their walking paths and this is the main cause of collision accidents. To minimize this problem, many researches develop technological solutions capable of detecting obstacles such as eletronic canes. However this type of solution is ineffective for different scenarios with limitations in the perception of obstacles, besides occupying one hand. This article present the development of a new solution wearable, that is the BlinGui for detecting static obstacles in indoor environments. Wearable devices have the advantage to function without the use of hands, and is observable and controllable. The evaluation had on average $90 \%$ positive results.
\end{abstract}

Resumo. Frequentemente os portadores de deficiência visual se deparam com objetos durante seus trajetos e esses são os principais causadores de acidentes por colisão. Para amenizar esse problema diversas pesquisas desenvolveram soluções capazes de detectar obstáculos, no entanto muitas como as que usam bengala são ineficazes para diversos cenários, com limitações na percepção de obstáculos, além de ocupar uma das mãos. Este artigo apresenta o desenvolvimento de uma nova solução vestível, que é o BlinGui para detecção de obstáculos estáticos em ambientes internos. Dispositivos vestiveis tem a vantagem de funcionar sem o uso das mãos, além de ser observável e controlável. A avaliação desta solução teve em média $90 \%$ de resultados positivos.

\section{Introdução}

Nos últimos anos a pesquisa científica tem criado métodos para facilitar o deslocamento dos Portadores de Deficiência Visual (PDV), que já são 285 milhões em todo o mundo segundo a Organização Mundial de Saúde [OMS 2016]. Esse número tem estimulado pesquisadores a usar tecnologias a favor desses indivíduos, que enfrentam diariamente grandes dificuldades em se locomover devido a existência de obstáculos não percebidos. Objetos estáticos como galhos de árvores, telefones públicos e fundo de caminhões podem prejudicar o deslocamento dos PDV como ilustra a Figura 1.

A bengala é uma ferramenta de baixo custo frequentemente utilizada por PDV como auxílio na locomoção, entretanto pode apresentar limitações como a falta de 


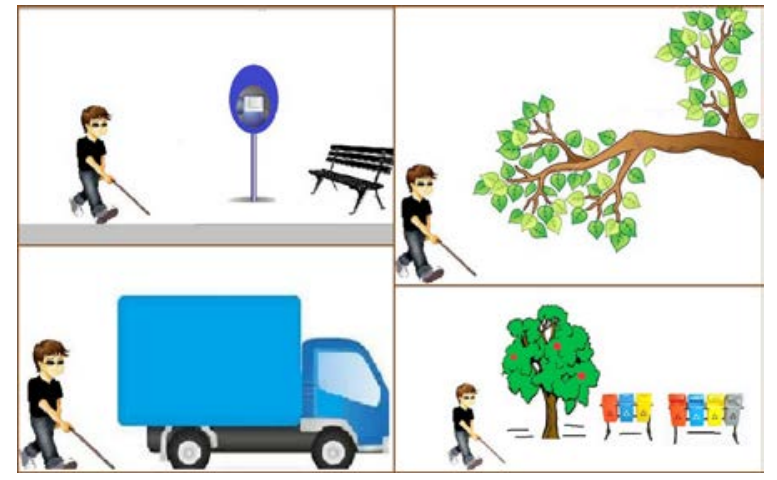

Figura 1. Cenários de interação entre PDV e obstáculos estáticos suspensos(imagens superiores) e rasteiros (imagem inferior).

percepção com objetos suspensos como, galhos de árvores e telefones públicos. Entretanto, existem soluções automatizadas acopladas a bengala [Kumar et al. 2014] e diversas outras complementares [Lee et al. 2013] que podem ser mais úteis. As chamadas bengalas eletrônicas detectoras de obstáculos que apesar de automatizadas continuam sendo uma bengala. Isso quer dizer que ainda possuem limitações, além de requerer exclusividade de uso das mãos do usuário. Todavia, existem as soluções complementares que são vestíveis e deixam o indivíduo com seus principais membros livres como boné eletrônico, cinto, mochila, colete, pulseira e colar. Essas soluções são capazes de se conectar ao usuário sem prejudicar suas atividades, não monopoliza a sua atenção e pode funcionar continuamente sem intervenções e ser controlada pelo usuário [Mann 1997].

O grande desafio observado nas soluções atuais é desenvolver ferramentas de baixo custo capazes de encontrar objetos imperceptíveis pela bengala. Assim como há muitas soluções aplicadas para detectar obstáculos, também existem diversos espaços para pesquisa nesta área. A detecção de objetos em um ambiente interno é um ponto que deve ser melhorado. Em vista disso, o objetivo geral deste trabalho é desenvolver uma solução vestível e acessível para detecção de obstáculos estáticos em ambientes interno, visando apoiar a locomoção dos PDV. Através de um protótipo eletrônico desenvolvido com as ferramentas advindas dos avanço da computação ubíqua. Uma vez que esta área promissora tem apresentado resultados de destaque no sentido da produção de protótipos computacionais miniaturizados, com boa capacidade de processamento e importantes características como mobilidade e transparência de localização [Weiser 1999]. Espera-se com essa pesquisa contribuir para os PDV com a prevenção contra colisões graves tornando a locomoção mais segura.

Além dessa introdução o restante deste artigo esta organizado como segue. A Seção 2 descreve a solução proposta, a Seção 3 apresenta a avaliação da solução, a Seção 4 traz a comparação entre os trabalhos correlatos e a proposta e a Seção 5 discute as conclusões e trabalhos futuros.

\section{BlinGui 2.0: Detecção de obstáculos}

Este trabalho propõe uma solução vestível para detecção de obstáculos em ambientes internos, visando apoiar a locomoção de PDV, deixando-os mais seguros dos caminhos a serem percorridos, conscientes dos obstáculos existentes e consequentemente mais integrados com o meio em que vivem. O intuito é que seja um recurso adicional para o usuário 
em combinação com os instrumentos rotineiros de locomoção (como a bengala). O nome BlinGui (lê-se blaingui) é oriundo do inglês Guide Blind que quer dizer guiar cegos. Essa solução está dividida em dois componentes (1) Guia e (2) Detecção de Obstáculos. O componente 2 será apresentado neste trabalho intitulado como BlinGui 2.0. A Figura 2 ilustra a arquitetura do BlinGui com seus componentes. O componente 1 é um aplicativo android que utiliza sinal Wi-Fi para localização e guia de PDV. Este pode ser utilizado em conjunto com o componente 2 que é um sistema vestível, uma pequena caixa de dimensões (103x130x50 mm) com itens eletrônicos embutidos e presa ao pescoço como um colar. Este é utilizado para detectar obstáculos por meio de sensor ultrassônico informando ao indivíduo sobre objetos em sua direção que podem causar colisão.

A ilustração da Figura 2 também destaca em duas dimensões uma pessoa PDV fazendo uso do protótipo. A imagem com a pessoa virada para o lado demonstra a varredura frontal que o sensor faz para capitar os obstáculos. A ilustração também redimensiona a representação do colar para uma melhor visualização. O sensor ultrassônico é apresentado acoplado com um servo motor, que faz a movimentação deste. Os dados que entram para serem processados são ilustrados como os valores das distâncias entre o dispositivo e um obstáculo. Estes dados são processados pelo arduíno e depois de toda lógica ser executada um sistema de alerta irá atuar com sons e luzes, as luzes são para PDV que distingue cores por vultos.

O sensor ultrassônico calcula a distância entre obstáculo e usuário através da emissão de ondas sonoras, a partir da distância estabelecida é possível detectar o obstáculo. Além da emissão de alertas o BlinGui 2.0 utiliza bluetooth, como ilustrado na Figura 2 para enviar informações a um smartphone que através de um aplicativo emite alertas de voz informando a direção do obstáculo (alto ou baixo) com vibrações.

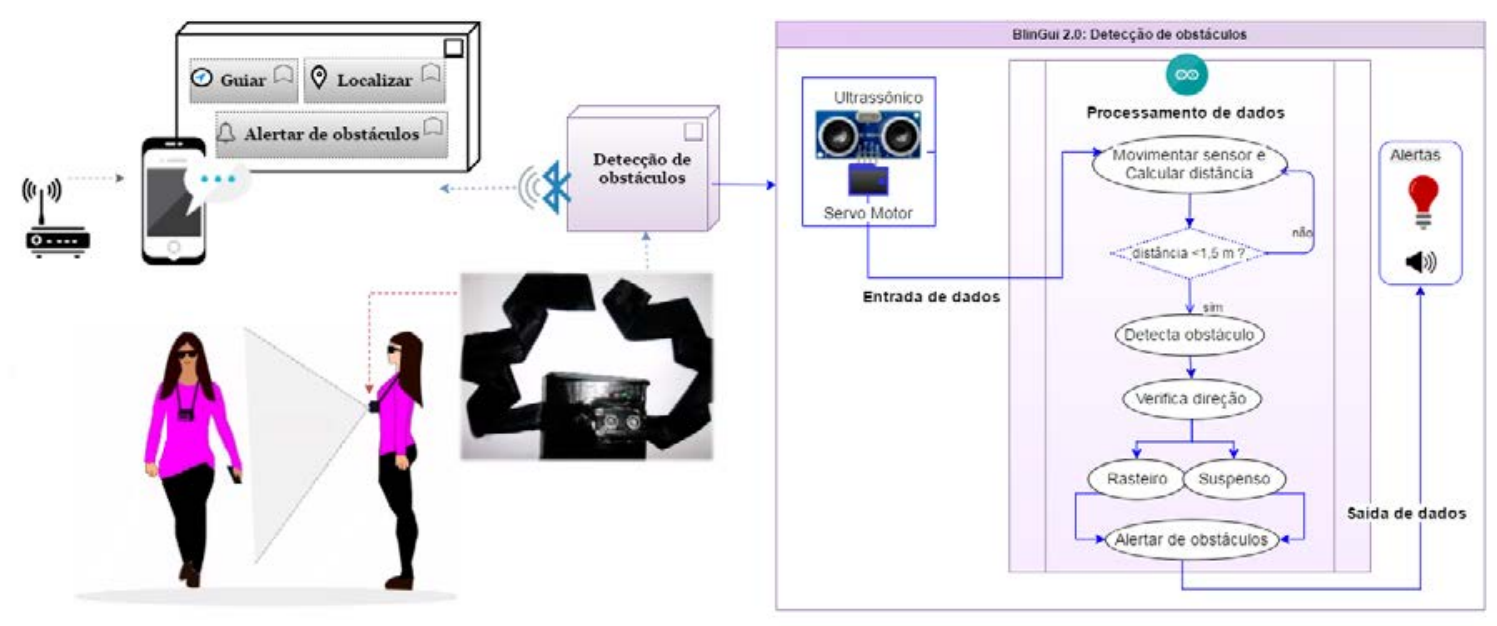

Figura 2. Arquitetura do BlinGui com o componente BlinGui 2.0 ilustrado em detalhes.

A Figura 3 ilustra o protótipo sendo utilizado por PDV em fase de testes com a detecção de obstáculos. O colar é composto por um Arduíno Uno, sensor ultrassônico, servo motor, módulo bluetooth, leds e buzzer. 


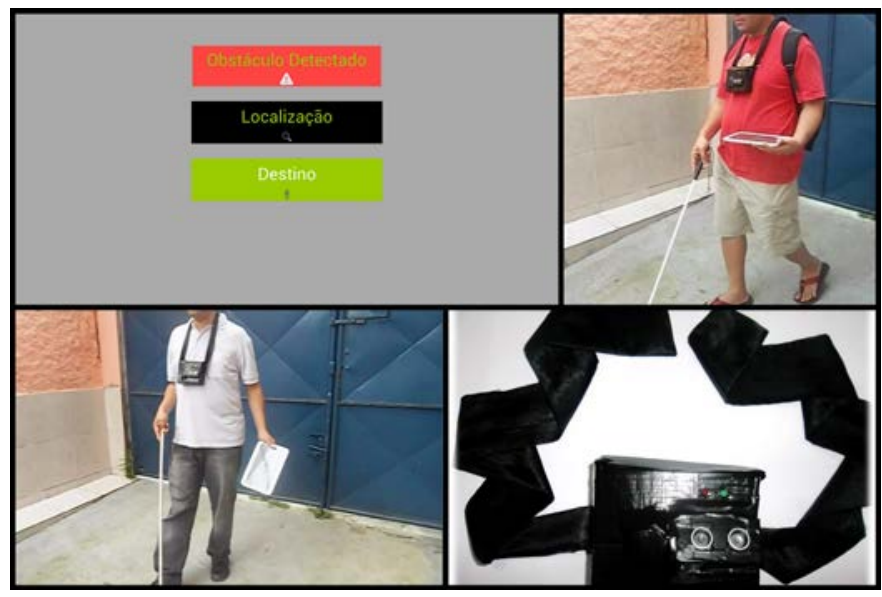

Figura 3. BlinGui sendo testado por PDV na Associação Baiana dos Cegos com detecção de obstáculos.

\section{Avaliação}

O ambiente utilizado para avaliação foi em uma Associação para PDV. No início deste trabalho um grupo de 12 indivíduos participou da pesquisa de campo respondendo a um questionário, cujo objetivo foi conhecer as especificidades do público alvo. Na fase final de avaliação um outro grupo de 8 indivíduos participou dos testes com o protótipo, alguns faziam parte do primeiro grupo, outros não, isso devido a alguns contratempos que impediram as mesmos indivíduos de estarem presentes.

O questionário aplicado tinham perguntas referentes ao tipo de deficiência de cada pessoa, as dificuldades mais comuns enfrentadas por eles diariamente, os principais obstáculos que mais provocavam colisões, se a bengala consegue detectar todos os obstáculos, quais tipos de alertas preferiam receber para avisar de obstáculos e se usariam um dispositivo preso ao corpo para ajudar na detecção. Todas as respostas foram registradas para serem analisadas. Os participantes envolvidos tinham entre 18 e 60 anos de idade e a maioria nasceu com a deficiência.

Para estimar o impacto do colar e sua aceitação por PDV, uma avaliação foi executada com 8 participantes com o objetivo de avaliar a seguinte hipótese:

- Hipótese: as colisões dos PDV com obstáculos estáticos durante um percurso são minimizadas com a utilização do BlinGui para detecção de obstáculos.

Espera-se que a hipótese seja aceita, de forma que um indivíduo ao fazer uso do BlinGui para detecção de obstáculos as colisões sejam minimizadas. Para isso foi conduzido um teste em uma área reservada de $200 \mathrm{~m}^{2}$, cujo objetivo era que os indivíduos usassem o dispositivo e trafegasse nesse espaço. Dessa forma poder avaliar se o indivíduo adaptou-se-se bem ao uso do dispositivo e se o dispositivo realizou as suas funções corretamente. Os testes foram divididos em duas etapas: Detecção de objetos rasteiros e Detecção de objetos suspensos. As duas etapas foram realizadas com e sem o colar eletrônico. Os indivíduos foram orientadas a percorrer uma trajetória com alguns obstáculos inseridos. Foram coletadas variáveis como tempo de cada percurso, obstáculos detectados corretamente e os não detectados. Os testes foram conduzidos com filmagem de cada etapa para uma posterior análise. 


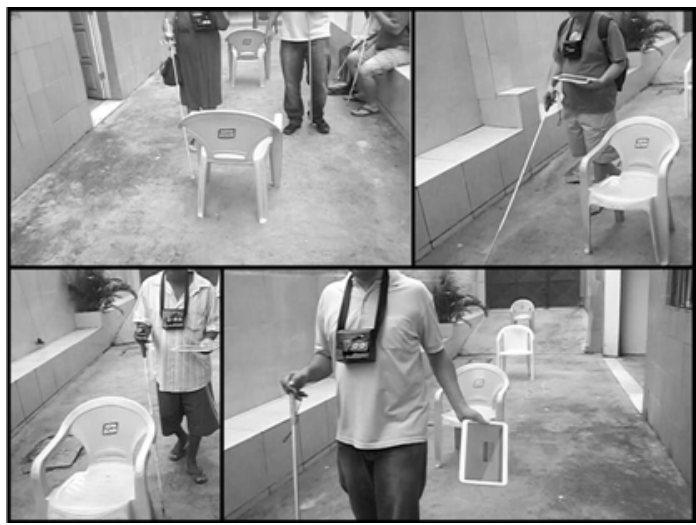

Figura 4. Execução dos testes para detecção de obstáculos rasteiros.

\subsection{Primeira Etapa da Avaliação - Detecção de Obstáculos Rasteiros}

Nesta etapa foram inseridos no caminho objetos rasteiros como cadeiras, lixeiras além das paredes e vasos que já continham no ambiente. Para cada objeto foi traçado um raio de $2 \mathrm{~m}$, com objetivo de verificar se o BlinGui 2.0 de fato emitia o alerta a $1,5 \mathrm{~m}$ do objeto. Estes testes foram feitos com e sem o uso do colar, nas duas situações os indivíduos preferiram utilizar a bengala. Porém o objetivo era que o protótipo alertasse dos obstáculos antes da bengala por mensagem de voz informando a direção do objeto. A Figura 4 exibe a realização desses testes.

Cada indivíduo fez um percurso com objetos espalhados no ambiente. A maioria dos obstáculos foi detectado de forma correta, porém teve momentos que a movimentação angular do sensor de distância era interrompida, ou tornava lenta. Nessas situações a detecção correta de obstáculos foi prejudicada, pois a direção do sensor poderia não estar voltada para o obstáculo principal a ser detectado. Um outro problema também encontrado com o dispositivo foi o excesso de alertas, que mesmo com o usuário parado ainda continuava informando excessivamente. Houve indivíduos não familiarizados com os alertas, e muitas vezes não se atentavam a tais informações e colidiram com os objetos.

\subsection{Segunda Etapa da Avaliação - Detecção de Obstáculos Suspensos}

Na segunda etapa dos experimentos bandeirolas suspensas na altura da cabeça do usuário foram inseridas no ambiente, simulando obstáculos suspensos para não provocar acidentes, já que a bengala não poderia ajudar neste caso. O objetivo era que o indivíduo percorresse o caminho com obstáculo e recebesse alertas com uma mensagem de voz sobre a direção do objeto detectado, se estar no alto ou baixo. Cada usuário executou este teste com e sem o uso do BlinGui, nos dois casos eles preferiram utilizar a bengala. A Figura 5 exibe a realização desses testes.

\subsection{Resultados}

Os resultados da pesquisa de campo constataram que todos os entrevistados concordam em utilizar o colar eletrônico de apoio à locomoção para ambientes internos não conhecidos, desses $90,0 \%$ acreditando que traria mais segurança no deslocamento. Todos os entrevistados relataram que já tiveram alguma espécie de colisão com objetos não detectados pela bengala, como fundos de caminhões, carros estacionados em locais indevidos, orelhões e postes.

Os resultados dos testes funcionais, para detecção de obstáculos rasteiros, resultaram na média de $88,0 \%$ detectados corretamente. Percebeu-se que com a movimentação 


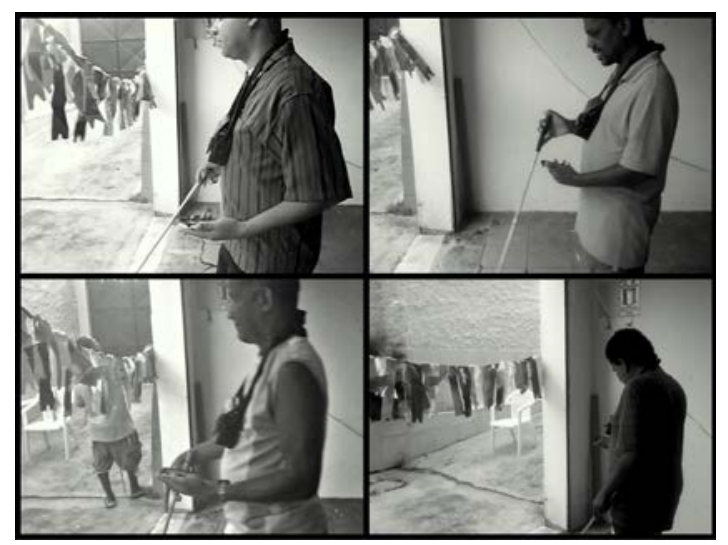

Figura 5. Execução dos testes para detecção de obstáculos Suspensos.

angular do sensor juntamente com o deslocamento do usuário o BlinGui 2.0 em casos específicos não identificou obstáculos corretamente. Nos testes executados sem o uso do colar sob as mesmas condições, foi observado que a bengala conseguiu detectar os mesmos obstáculos, porém quase colidindo. O BlinGui alertava com 1,5m do obstáculo, deixando o usuário atento sobre possíveis colisões.

Os testes executados para obstáculos suspensos resultaram na média de 90,0\% detectados corretamente, o restante foram alertados com alguns atrasos, ou não alertou corretamente. Em todas as execuções dos testes a bengala não detectou os obstáculos suspensos, pelo fato do obstáculo estar a uma altura superior, o que torna difícil a detecção. A autonomia do protótipo foi satisfatória na execução dos testes com uma duração média da bateria. Baseados nesses testes iniciais o BlinGui 2.0 apresentou resultados satisfatórios com relação a detecção de obstáculos. A média e o intervalo de confiança foram calculados para cada um dos testes, considerando uma confiança de 95,0\%. Os valores são representados na Tabela 1.

Tabela 1. Intervalo de Confiança dos Testes

\begin{tabular}{|l|l|l|l|}
\hline \multicolumn{1}{|c|}{ Testes } & Média & \multicolumn{1}{c|}{ Desvio Padrão } & Intervalo de Confiança \\
\hline \hline Obstáculos Rasteiros & 4,4 & 0,55 & {$[4,38 ; 4,41]$} \\
\hline Obstáculos Suspensos & 1,8 & 0,45 & {$[1,78 ; 1,81]$} \\
\hline
\end{tabular}

Fundamentado nos testes executados pode-se aceitar a hipótese, com 95,0\% de confiança, que as colisões dos PDV com obstáculos estáticos durante um percurso são minimizadas com a utilização do BlinGui 2.0. Em relação ao tempo não houve diferenças devido ao lugar ser um ambiente já conhecido pelos usuários. Pelo fato do colar ser algo novo para eles, alguns acabaram se distraindo com os alertas e avisos sonoros, ocasionando uma diminuição dos passos comprometendo uma amostragem real do tempo, já os demais permaneceram com passos normais. Opiniões dos usuários foram analisadas após a execução dos testes para verificar a aceitação do BlinGui 2.0, e todos concordaram em utilizar o colar eletrônico no dia-a-dia por ser confortável e leve, além de detectar objetos que muitas vezes não são encontrados pela bengala. Os hardwares utilizados para compor o colar têm alta disponibilidade no mercado e são de baixo custo, a fim de que o preço seja acessível para o usuário.

\section{Comparação entre o BlinGui 2.0 e alguns Trabalhos Correlatos}

As soluções correlatas apresentadas neste trabalho se baseiam nas vestíveis, acopladas à bengala e móveis. A Figura 6 exibe alguns trabalhos relacionados e a Tabela 2 apresenta 
a comparação entre eles. Para as soluções que têm um dispositivo acoplado a bengala foram identificadas algumas desvantagens, como:

- Limitação aos movimentos: obstáculos só serão encontrados onde a bengala for posicionada, por exemplo um suporte de telefone público é de difícil percepção através da bengala.

- Não vestível: O usuário só conta com a utilização de uma das mãos para suas demais atividades, pois a outra está exclusiva para a bengala.

- Dependência: priva o indivíduo de ficar independente da bengala em algum momento de sua vida, mesmo com o uso da tecnologia que deveria propiciar novas alternativas.

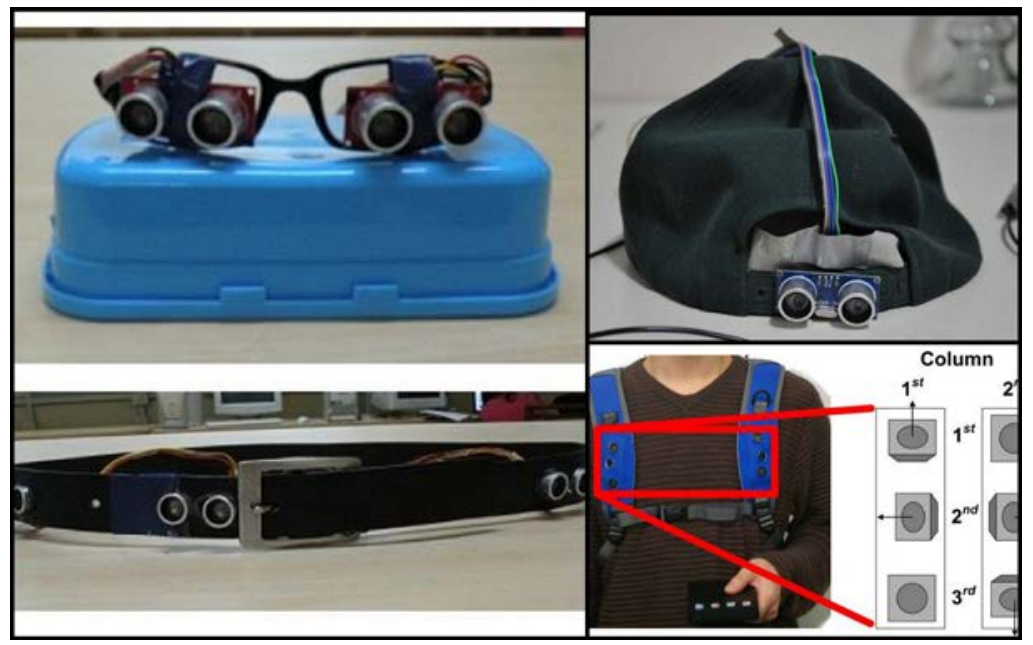

Figura 6. Algumas Soluções de apoio à locomoção de PDV vestíveis.

Tabela 2. Relação entre os trabalhos existentes na literatura e a solução proposta

\begin{tabular}{|c|c|c|c|c|c|c|c|}
\hline $\begin{array}{c}\text { Soluções } \\
\text { Correlatas }\end{array}$ & Descrição das Soluções & Vestível & $\begin{array}{l}\text { Bengala } \\
\text { Acoplada }\end{array}$ & Mobile & $\begin{array}{l}\text { Aéreo e } \\
\text { Rasteiro }\end{array}$ & Externo & Interno \\
\hline [Long et al. 2010] & $\begin{array}{l}\text { Sistema de obstáculos baseado em visão } \\
\text { estéreo combinado com atenção visual. }\end{array}$ & $?$ & $?$ & $?$ & $\checkmark$ & $\sqrt{ }$ & $\sqrt{ }$ \\
\hline [Garcia et al. 2011] & $\begin{array}{l}\text { Bengala eletrônica que detecta obstáculos } \\
\text { acima da cintura, utiliza sensor ul- } \\
\text { trassônico e alertas vibratórios. }\end{array}$ & $x$ & $\sqrt{ }$ & $x$ & $x$ & $\sqrt{ }$ & $\sqrt{ }$ \\
\hline $\begin{array}{l}\text { [Hernandez et al. } \\
\text { 2012] }\end{array}$ & $\begin{array}{l}\text { Usa estéreo visão, Kinect e regras de de- } \\
\text { cisão fuzzy. }\end{array}$ & $\sqrt{ }$ & $x$ & $x$ & $\sqrt{ }$ & $x$ & $\sqrt{ }$ \\
\hline $\begin{array}{l}\text { [Bhatlawande et } \\
\text { al. 2012] }\end{array}$ & $\begin{array}{l}\text { Cinto e óculos eletrônico utiliza sensor ul- } \\
\text { trasônico. }\end{array}$ & $\sqrt{ }$ & $x$ & $x$ & $x$ & $\checkmark$ & $\checkmark$ \\
\hline [Savvas et al. 2012] & $\begin{array}{l}\text { Luva eletrônica composta de ultrassônico e } \\
\text { alertas vibratórios, sonoros e Bússola Dig- } \\
\text { ital. }\end{array}$ & $\sqrt{ }$ & $x$ & $x$ & $x$ & $\checkmark$ & $\sqrt{ }$ \\
\hline [Lee et al. 2013] & $\begin{array}{l}\text { Mochila inteligente possui sensores ul- } \\
\text { trassônicos nas alças em vários ângulos, } \\
\text { usa redes zigbeen. }\end{array}$ & $\checkmark$ & $x$ & $\sqrt{ }$ & $\checkmark$ & $\sqrt{ }$ & $x$ \\
\hline [Kumar et al. 2014] & $\begin{array}{l}\text { Dispositivo acoplado a bengala, } \\
\text { informações de obstáculos pelo colete. }\end{array}$ & $\sqrt{ }$ & $\checkmark$ & $x$ & $\sqrt{ }$ & $\sqrt{ }$ & $\sqrt{ }$ \\
\hline [Rey et al. 2015] & $\begin{array}{l}\text { Boné eletrônico utiliza sensor ultrassônico } \\
\text { e arduíno, detecta obstáculos acima da } \\
\text { cabeça. }\end{array}$ & $\checkmark$ & $x$ & $x$ & $x$ & $\sqrt{ }$ & $\checkmark$ \\
\hline $\begin{array}{l}\text { BlinGui } 2.0 \\
\text { (Proposta) }\end{array}$ & $\begin{array}{l}\text { Colar eletrônico detecta obstáculos rasteiro } \\
\text { e suspensos em ambientes internos. Utiliza } \\
\text { ultrassônico e arduíno e um smartphone. }\end{array}$ & $\checkmark$ & $x$ & $\sqrt{ }$ & $\checkmark$ & $x$ & $\sqrt{ }$ \\
\hline
\end{tabular}


Alguns trabalhos como por exemplo, Electronic Long Cane (ELC) [Garcia et al. 2011] e Ultrassom Cane [Kumar et al. 2014] decidiram automatizar o instrumento rotineiro utilizado pelos PDV. As soluções desenvolvidas foram acopladas à bengala para encontrar obstáculos de difícil acesso. A proposta do brasileiro Garcia é uma bengala eletrônica que sinaliza obstáculos acima da cintura. A proposta de Kumar é mais completa detectando obstáculos em outras direções e buracos, utiliza um colete pra receber avisos vibratórios e sonoros. Os dois realizaram experimentos, Kumar com dez voluntários vendados, Garcia com oito PDV. Os dois trabalhos não deram resultados que provem estatisticamente a validade das soluções.

Obstacle Detection Stereo vision [Long et al. 2010] e Cognitive Guidance [A. Landa-Hernandez 2012] utilizam visão computacional para detecção de obstáculos. Na proposta de Long não ficou claro se o sistema é vestível, porém a de Hernandez ficou explícito que é um protótipo em fase de construção. Apesar de vestível como uma espécie de capacete precisa carregar sempre um computador nas costas. Os dois declaram ter feito testes de avaliação porém, Hernandez não demonstrou resultados por meio de dados que validasse a sua proposta. Long apresentou uma tabela com alguns resultados que não foram analisados no artigo. Os dois não realizaram testes com PDV.

Waist-Belt for Visually Impaired [Bhatlawande et al. 2012] é um cinto e óculos detector de obstáculos composto com pares de sensores ultrassônicos, e Smart Backpack [Lee et al. 2013] é a mochila inteligente com sensores ultrassônicos acoplados em cada alça e um computador portátil. A solução de Bhatlawande detecta obstáculos na altura da cintura e cabeça, enquanto que a de Lee é mais completa calcula a posição do usuário, avalia a localização de objetos e determinar a direção da caminhada. Os testes feitos para avaliar o cinto e óculos foram executados com quatro usuários PDV treinados e quatro inexperientes. Segundo autor os resultados comprovaram uma boa precisão do dispositivo em descobrir obstáculos. Os testes de Lee com quatro pessoas de olhos vendados teve taxa de sucesso maior do que $95 \%$.

Os dois últimos trabalhos correlatos são o CYCLOPS [Savvas et al. 2012] e o Blind Guardian [Rey et al. 2015]. O CYCLOPS, luva eletrônica, é composto de um sensor ultrassônico, uma bússola digital com acelerômetro e motor de vibração. O Blind Guardian é um boné que detecta obstáculos na altura da cabeça, o alerta é dado por vibração na mão direita do usuário. Enquanto as luvas ocupam um membro do corpo o boné possibilita liberdade as mãos. Porém não se pode dizer qual dos dois é mais útil, pois os resultados dos testes não possibilitam essa comparação. A avaliação da luva foi feita com um total de 16 testadores PDV e vendados. O Blind Guardian fez os testes com 29 indivíduos vendados onde $68,9 \%$ não colidiram com os obstáculos.

A Tabela 2 traz comparações dos trabalhos com o BlinGui 2.0, suas características, funcionalidades e tecnologias por ordem cronológica. A maioria dos trabalhos são sistemas vestíveis, os demais são acoplados a bengala ou ao computador, com detecção de obstáculos suspensos e rasteiros. Muitos trabalhos deixam a entender que funcionam em espaços internos e externos. Porém não houve relatos se aplicaram técnicas variadas para a detecção de obstáculo em ambientes distintos, visto que existem características diferentes para esses ambientes. Os trabalhos também não deixaram claro se as soluções detectam objetos em movimento com a mesma precisão dos estáticos. Esta pesquisa é ciente de que existem diferenças na detecção de obstáculos estáticos ou em movimentos 
em ambientes internos ou externos, com isso deixa bem explícito em que domínio está inserida.

O BlinGui 2.0 possui independência da bengala, enquanto as soluções que se acoplam a ela, como as de Garcia [Garcia et al. 2011] e Kumar [Kumar et al. 2014] não possuem tal independência. Por ser uma solução vestível o colar está sempre conectado com o usuário, alguns trabalhos porém não se atentam a esta contribuição, como a solução de Long [Long et al. 2010]. À medida que o BlinGui é pequeno (103x130x50 mm), leve (140 gramas aproximadamente) e não proporciona desconforto, a proposta de Hernandez [A. Landa-Hernandez 2012] faz o usuário carregar alguns hardwares pesados sobre o corpo que pode atrapalhar a locomoção, a solução de Bhatlawande [Bhatlawande et al. 2012] possui mais de um dispositivo, e a mochila de Lee [Lee et al. 2013] pode ser grande e desconfortável ao usuário que deverá sempre carregála. O BlinGui utiliza somente um colar que permite as mãos do usuário ficarem livres, o que não acontece na luva de Savvas [Savvas et al. 2012]. O boné [Rey et al. 2015] só detecta objetos na altura da cabeça, enquanto o BlinGui em mais de uma direção.

\section{Conclusões e Trabalhos Futuros}

Colisões frequentes com obstáculos são reais problemas enfrentados pelos PDV, que sofrem diariamente com a possibilidade de danos fatais ou permanentes. Como exemplo, a perfuração de um dos olhos, causado pela colisão com o fundo de um caminhão estacionado em um local indevido, caso verídico relatado por um PDV. Baseado nessa problemática esse trabalho se propôs a contribuir com uma nova solução capaz de alertar ao usuário sobre possíveis colisões, evitando esses indivíduos passarem por situações temerosas como esta.

Este artigo popôs o BlinGui 2.0 como uma solução para detecção de obstáculos estáticos em ambientes internos, representado como colar eletrônico vestível, que detecta obstáculos suspensos e rasteiros. A solução foi desenvolvida e testada, os testes foram feitos com os PDV e estimaram uma média de quase $90 \%$ que o colar funciona satisfatoriamente. Os testes foram feitos em situações específicas, mas novas avaliações deverão ser feitas como trabalhos futuros, afim de testar todas as possibilidades possíveis, com variedades de objetos e em locais diferentes. Através dos testes foram reveladas algumas falhas de precisão existentes no colar. Aumentar a precisão dos métodos de Detecção de obstáculos é uma questão de muita relevância para próximas pesquisas.

Esse trabalho deixou várias lacunas que possibilitam o desenvolvimento de novos trabalhos. Uma dessas é integrar a computação sensível ao contexto [Vieira et al. 2009] ao BlinGui 2.0, para a detecção de obstáculos estáticos em qualquer ambiente com adaptação ao contexto do usuário. Projeto este que já está em fase de desenvolvimento, e será a próxima versão desse trabalho.

Os trabalhos correlatos levantaram a seguinte discussão: não deixaram claro o domínio ao qual estão inseridas suas pesquisas. Pelo fato, de não informarem se as ferramentas desenvolvidas são para qualquer ambiente(internos ou externos) e para qualquer obstáculo(estático ou em movimento). Esses critérios não foram evidenciado, deixando a entender que em alguns casos a solução engloba todo esse domínio. Porém isso só poderá ser comprovado por diversos testes aplicados em várias dessas situações, deixando claro as técnicas utilizada que resolvem esse problema. 


\section{References}

A. Landa-Hernandez, E. B.-C. (2012). Cognitive guidance system for the blind. World Automation Congress (WAC), 2012, pages 1-6.

Bhatlawande, S., Mukhopadhyay, J., and Mahadevappa, M. (2012). Ultrasonic spectacles and waist-belt for visually impaired and blind person. National Conference on Communications, pages 8-11.

Garcia, A., Fonseca, R., and Duran, A. (2011). Electronic long cane for locomotion improving on visual impaired people. a case study. IEEE Pan American Health Care Exchanges.

Krumm, J. (2009). Ubiquitous Computing Fundamentals. Chapman \& Hall/CRC, 1st edition.

Kumar, K., Champaty, B., Uvanesh, K., Chachan, R., Pal, K., and Anis, A. (2014). Development of an ultrasonic cane as a navigation aid for the blind people. IEEE, International Conference on Control, Instrumentation, Communication and Computational Technologies.

Lee, J. H., Kim, K., Lee, S. C., and Shin, B. S. (2013). Smart backpack for visually impaired person. Proceedings - International Conference on ICT for Smart Society: "Think Ecosystem Act Convergence", pages 26-29.

Long, C., Bao-long, G., and Wei, S. (2010). Obstacle Detection System for Visually Impaired People Based on Stereo Vision. Fourth International Conference on Genetic and Evolutionary Computing, pages 723-726.

Mann, S. (1997). Wearable computing: A first step toward personal imaging. Computer, 30:25-32.

OMS (2016). Organização mundial de saúde: Cegueira e deficiência visual. Disponível em: http://www.who.int/mediacentre/factsheets/fs282/es/.

Rey, M., Hertzog, I., Kagami, N., and Nedel, L. (2015). Blind guardian: A sonar-based solution for avoiding collisions with the real world. XVII Symposium on Virtual and Augmented Reality.

Savvas, D., Salonikidou, B., Diamantis, G., and Astaras, A. (2012). Development and evaluation of an open source wearable navigation aid for visually impaired users (CYCLOPS). IEEE 12th International Conference on BioInformatics and BioEngineering, pages $115-120$.

Vieira, V., Tedesco, P., and Salgado, C. (2009). Modelos e Processos para o Desenvolvimento de Sistemas Sensíveis ao Contexto. Challenges, 20:381-431. Disponível em: http://homes.dcc.ufba.br/ vaninha/context/2009_TextoJAI_Final.pdf.

Weiser, M. (1994). The world is not a desktop. Interactions, 1(1):7-8.

Weiser, M. (1999). The computer for the 21st century. SIGMOBILE Mobile Computing and Communication Review, 3(3):3-11. 